\title{
Historische Kontingenz und universale Relevanz in neueren Prozeß-Christologien*
}

\author{
Prof. Dr. Hans Schwar z, Rilkestr. 44, 8411 Lappersdorf
}

John W. Wires hat vielleicht sein Argument etwas überzogen, wenn er behauptete: "Zum ersten Mal seit dem 13. Jahrhundert wird ein selbstbewußter Versuch gemacht, die Struktur der christlichen Theologie auf dem Fundament einer rationalen Metaphysik zu errichten. ${ }^{1}$ In der Tat setzt die Prozeßtheologie eine rationale Metaphysik voraus. Deshalb ist es nicht überraschend, daß Prozeßtheologen gerne über Gott reden, aber nur zögernd über Christologie und den Stellenwert der Christologie für die Theologie insbesondere. Wenn wir ein Buch wie David Griffin's Process Christology lesen, bekommen wir den Eindruck, daß die christologische Reflexion einem eindrucksvollen theologischen Diskurs gleichsam nur angefügt worden ist. Der Grund für diese Vernachlässigung der Christologie scheint in der Spannung zwischen historischer Kontingenz und universaler Relevanz zu liegen. Letztere scheint die erstere soweit zu verdrängen, daß Jesus Christus höchstens ein Modell für Gottes Handeln mit der Welt abgibt. Aufgrund einer Metaphysik, die durch die Vernunft errichtet und verteidigt wird, ist das Neue, welches solch ein Modell einführen könnte, auf die der Welt eigenen Möglichkeiten beschränkt. Im folgenden wollen wir neuere christologische Entwürfe dreier bekannter Prozeßtheologen, Norman Pittenger, John B. Cobb und David Griffin kritisch analysieren, um zu sehen, wie die Spannung zwischen historischer Kontingenz und universaler Relevanz bestimmt wird.

\section{Norman Pittenger}

In Christology Reconsidered (1970) behauptet Norman Pittenger gleich zu Anfang, daß kein geschichtliches Einzelwesen unter Absehung von dem Kontext, in dem es erscheint, verstanden oder interpretiert werden kann. Dies trifft auch auf Jesus zu, der ein Jude im tiefsten Sinne war. Seine Jünger fühlten eine tiefe Hingabe und Loyalität zu ihm. Aber wer war und ist dieser Jesus?

Im Exzerpt vorgetragen und diskutiert in der "Currents in Contemporary Christology" Gruppe auf der Jahrestagung der American Academy of Religion, Chicago, 8.-11. Dezember 1984.

John W'. Wires, “The Search for the Christological Jesus”, St. Luke's Journal of Theology 21 (Dezember 1977): 19. Es ist nicht unwichtig, daß die vorzügliche Arbeit von Michael Welker, Universalität Gottes und Relativität der Welt, Neunkirchen-Vluyn 1981, sich bei der Untersuchung des Prozeßdenkens folgerichtig hauptsächlich mit theologischer Kosmologie befaßt. 
Nach Pittenger kann Jesus ohne einen theistischen Bezug nicht sachgemäß verstanden werden. Zunächst muß man jedoch sehen, daß Jesus ein Mensch im vollen Sinne war. In "dem historischen und menschlichen Ereignis Jesus Christus, der ein Mensch unter Menschen war, zu einer bestimmten Zeit und an einem bestimmten Ort, ergab sich eine Wirksamkeit Gottes, die durch eine besondere Qualität bestimmt ist und ein entscheidendes Moment in der langen Geschichte Gottes mit seiner Welt darstellt. $\star^{2}$ In Jesus Christus trafen sich das Menschliche und Göttliche in einer solchen Weise, daß »es eine Einheit der menschlichen und göttlichen Tätigkeit gab. ${ }^{3}$ Durch einen wirklichen Menschen ist Gott so am Werk, daß Gottheit und Menschheit in ihm wirklich ständig und integral und in der ganzen Tätigkeit eins sind, die wir benennen, wenn wir Jesus Christus sagen. Doch die sich wechselseitig durchdringende Tätigkeit Gottes, nämlich seine inkarnierende Bewegung, vollzieht sich im ganzen Kosmos. Wenn Pittenger über die Letztlichkeit der Tätigkeit Gottes in Jesus Christus redet, will er damit nicht sagen, daß Gott seitdem aufgehört hat, tätig zu sein. Gott ist durch seine Natur unaufhörlich schöpferisch und aktiv in der ganzen Welt am Werk.

Pittenger schließt nicht aus, daß es auch andere "at-one-ings " (vereinigende Versöhnungen) gibt. Doch kennt er die Kirche als Gottes Weise an, "um nachfolgenden Generationen das anzubieten, was er in Jesus wirkte und deshalb integral für das ganze Ereignis ist, welches wir Jesus Christus nennen «. Gott wählte diese Weise, um seine Menschenkinder durch die Qualität, den Charakter und den besonderen Geist, der das Ergebnis des Lebens Jesu Christi in der Welt ist, zur Einheit mit ihm zu bringen. Die Bejahung des entscheidenden Charakters Jesu Christi im Erlösungsprozeß kann man nicht von einem demonstrierbaren Faktum ableiten, sondern sie muß aus einer bestimmten Glaubenshaltung heraus kommen. Wir können nicht mit einer vergleichenden Studie der Religionsgeschichte argumentieren, da wir keinen einzelnen Aspekt einer Religion isolieren können, einschließlich ihres entscheidenden Charakters, um ihn zu vielleicht ähnlichen Aspekten in anderen Religionen in Beziehung zu setzen, ohne daßs man ihn im Kontext aller anderen Dinge in dieser Religion betrachtet.' Zudem können wir keine neutrale, objektive Haltung gegenüber religiösen Phänomenen einnehmen und dann noch einfühlend genug sein, um das tief genug zu durchdringen, was wir untersuchen. Wenn wir uns mit einer Religion einlassen, haben wir nicht mehr genügend Abstand zu einem. objektiven Vergleich.

\footnotetext{
2 Norman Pittenger, "The Incarnation in Process Theology", Review and Expositor 7: (Winter 1974): 48.

3 Ebd.

4 Norman Pittenger, Christology Reconsidered, London 1970, 96.

s So Pittenger, ebd., 103.
} 
Wie können wir im Lichte dieser Problematik die universale Relevanz Christi bejahen, ohne anderen Religionen Unrecht zu tun? Pittenger findet eine Lösungsmöglichkeit in Person und Werk Jesu Christi. Nach dem Prozeßdenken ist die Liebe das grundlegende Motiv Gottes und seiner Schöpfung. "Gott ist Liebe; er ist der kosmisch Liebende «'. Für Prozeßtheologen ist Liebe "keine sentimentale Haltung, sondern ein positives Geben und Nehmen, ein Miteinanderteilen, Ekstase und Furcht, wie es sich in Jesu eigenem Kontakt mit anderen zeigt, in seinem ganzen Lehren und in dem Verständnis, das seinen tiefsten Ausdruck am Kreuz findet. ${ }^{7} \mathrm{Da}$ in Gott Sein und Akt zusammenfallen, ist die Liebe dort in vorauslaufender Weise am Werk, wo und wann sich eine Antwort auf die göttliche Liebe zeigt. Jesu Einzigartigkeit ist nicht exklusiv, sondern schließt alles ein, was anderswo von Gott offenbart ist. Jesus Christus ist die besondere, entscheidende und definitive Offenbarung Gottes, da er offenbart, "was Gott wirklich als Gott ist « ${ }^{8}$. Er ist der menschlich vermittelte Ausdruck der Liebe, die Gott in sich selbst ist.

Pittenger folgert daraus, daß »es unbegreiflich ist, daß er [Gott] jene zur ewigen Verbannung von seiner Gegenwart verdammen sollte, die ihn ohne eigene Schuld in jener Vermittlung nicht haben erkennen können, oder die, die von ihm zwar gehört haben, aber aus irgendeinem Grund die gehörte Botschaft unmöglich haben annehmen können. "' Gott wird niemals davon ablassen und damit aufhören, seine Kinder zu suchen und zu finden. Es ist Gottes Aufgabe, die Erlösung der Menschheit möglich zu machen, und nicht die der Kirche durch ihre Verkündigung. Die Kirche muß jedoch verkündigen, daß der Gott, dem niemand entkommen kann, der gleiche ist, der seine Liebe in Jesus Christus erklärte. Pittenger proklamiert keinen seichten Universalismus. Im Gegenteil gesteht er zu: „Was Gott mit denen vorhat, die nicht von Jesus Christus gehört haben, ist uns nicht offenbart. ${ }^{10}$ Wir müssen alle, auch uns selbst, den Händen Gottes anvertrauen. "Aber der Christ weiß oder sollte wissen, daß dies liebende Hände sind. "1"

Obwohl Pittenger die historische Kontingenz Jesu Christi bejaht, behauptet er auch seine universale Relevanz. Während er ein sachgemäßes Verhältnis zwischen beiden aufrechterhält, sollten wir auch erkennen, was bei ihm unerwähnt bleibt. Was ist etwa Gottes Antwort auf jene, die beharrlich sich weigern, ihn als einen Gott der Liebe anzunehmen? Wir sollten nicht vergessen, daß die Inkarnation der Liebe Gottes in unserer

\footnotetext{
"Norman Pittenger, "The Incarnation in Process Theology", 51.

'Norman Pittenger, "Christology in Process Theology", Theology 80 (Mai 1977): 189.

"Norman Pittenger, "The Incarnation in Process Theology", 56.

"Norman Pittenger, Christology Reconsidered, 106.

ic Ebd., 107.

"Ebd.
} 
dunklen Welt starb. Hier wurde die Beachtung des Sabbats von frommen Menschen für wichtiger erachtet als der Erweis der Liebe durch Gottes heilende Kraft. Zum Schluß verursachten solche Menschen auch Jesu Tod. Dann gab es die rücksichtslosen Römer und einen opportunistischen Prinzling. Diese haben auch heute noch Nachfolger. Sind sie aber wirklich so verbesserungsfähig, daß sie schließlich von dem kosmisch Liebenden überzeugt werden? Die theoretische Konstruktion eines alle überzeugenden kosmisch Liebenden steht im starken Kontrast zu den historischen Wirklichkeiten von Auschwitz, Hiroshima und der Ermordung von Erzbischof Romero. Die universale Relevanz Christi kann man nicht mit der Behauptung bekräftigen, daß sich Gottes Liebe in der rätselhaften Polarität von Kreuz und Auferstehung ausdrückt. Nur nachdem das Tal des Todes durchschritten wurde, kam die Hoffnung der Auferstehung.

\section{John B. Cobb, Jr.}

Bevor er seine umfangreiche Christologie, Christ in a Pluralistic Age (1975) veröffentlichte, hatte John Cobb die Frage nach letzter Verbindlichkeit Christi schon einige Male angeschnitten. In einem Aufsatz von 1966 behauptete Cobb, daß "Jesus uns radikal zeigt, was es bedeutet, von Gott her und für Gott hin zu existieren. Wir sind unfähig, uns eine vollständigere Verkörperung dieser Existenzweise vorzustellen und die Geschichte hat uns sicher bis jetzt noch keine angeboten. Wo wir der stärksten Annäherung an diese Daseinsweise begegnen, finden wir auch jene, die ihre Abhängigkeit von Jesu betonen müssen und zugleich ihre Distanz von der Verkörperung dieser Existenz. Obwohl wir als Historiker nicht behaupten können, daß Jesu Verwirklichung in der Zukunft nicht erreicht werden wird, ist es höchst unwahrscheinlich, daß eine Annäherung daran seinem Einfluß gleichkommen wird. Deshalb wird solch eine Möglichkeit die letzte Verbindlichkeit Jesu nicht infrage stellen ${ }^{12}{ }^{12}$

Die Besonderheit Jesu zeigt sich für Cobb darin, wie sich Jesus verhält und wie andere, die ihm dabei nachfolgen wollen, ihn nicht erreichen können. Sein Verhalten wird dann als schlechthinnige Existenz von Gott und zu Gott interpretiert. Wenn Sein und Akt identisch sind, würde das bedeuten, daß Jesus schlechthin als Gott existiert. Doch im Gegensatz zu Pittenger setzt Cobb beide nicht explizit gleich. Im Kontrast zu Pittenger will Cobb auch, "zumindest am Anfang, die letzte Verbindlichkeit Jesu und die christliche Kirche " radikal trennen. ${ }^{13}$ So kann er behaupten, daß die letzte Verbindlichkeit, die sich in Jesu Existenzweise anzeigt, auch außerhalb des christlichen Bereichs zum Ausdruck kommt, z. B. wenn Hindus

\footnotetext{
${ }^{12}$ John B. Cobb, "The Finality of Christ in a Whiteheadian Perspective", in The Finality of Christ, hg. v. Don Kirkpatrick, New York 1966, 132.

13 Ebd., 137.
} 
ohne aufzuhören Hindus zu sein, in Jesus die Erfüllung des Hinduismus finden. Damit führt Jesus einen Hindu zu einer »schöpferischen Umwandlung" (creative transformation) seines eigenen Glaubens, ein Begriff, der von Cobb später weiter benutzt wird.

Es ist bedeutsam, daß Cobb von Jesus spricht und daß man in Jesus die Erfüllung findet, denn er vermeidet bewußt den Begriff Jesus Christus. Doch müssen wir hier noch fragen, ob man denn die universale Bedeutung Jesu hinreichend verstehen kann, wenn man ihn nicht als den erkennt, der in der Tat der Christus ist. Solche Anerkennung ist untrennbar mit dem historischen Phänomen der jüdisch-christlichen Tradition verknüpft und kann ohne sie nicht adäquat vollzogen werden. Cobb jedoch will seine Anerkennung Jesu von dieser Tradition abtrennen. Der Grund liegt darin, daß Cobbs Argumentationsweise viel axiomatischer als historisch ist. Dies wird wiederum deutlich, wenn er betont: »Wenn für letztliche Gültigkeit argumentiert werden soll, dann muß sie in den Konsequenzen seiner Gegenwart für unsere Existenz und besonders in den Konsequenzen für unser Verhältnis zu Gott aufgewiesen werden. «"

In einem Aufsatz von 1971 über die Whitehead'sche Christologie zieht John Cobb die historischen Linien weiter aus. Er vergleicht Jesus mit den israelitischen Propheten und behauptet: "Das Ich Jesu, statt sich mit dem göttlichen Ich zu vergleichen, identifizierte seine Autorität mit der Gottes. Unter den religiösen Führern der Menschheit ist dies einzigartig. « ${ }^{15}$ Indem Cobb von der historischen Besonderheit her argumentiert, kann er an der Einzigartigkeit Jesu festhalten. Er geht sogar auf die Entstehungsphase des christologischen Dogmas ein und stellt fest, daß das Ich Jesu weder mit dem göttlichen Ich zusammenfloß noch durch es ersetzt wurde. Es behielt jedoch seine autonome Existenz, indem es sein Verstehen mit dem Gottes identifizierte. Der eine Gott war in einzigartiger Weise in Jesus gegenwärtig, da in ihm kein Aspekt seiner Menschheit durch Gott ersetzt wurde. Jesus war dieser göttlichen Gegenwart in ihm gewahr, da er nicht nur über Gott sprach, sondern ihn verkörperte, indem er von und zu Gott lebte. Er war, was er sagte und tat.

Was hat jedoch Jesus tatsächlich durch seine Einzigartigkeit bewirkt? Es ist interessant, daß John Cobb nicht versucht, die erlösende Bedeutung Jesu aufzuweisen, sondern stattdessen bekennt: "Es ist meine Überzeugung, daß Jesus für jene, die auf ihn antworteten, eine letztliche und unübertreffliche Struktur der Existenz ins Dasein brachte.... Als Erlöser ist Jesus nicht nur der Grund der neuen Existenzstruktur, sondern auch der, in Beziehung zu dem die Gesundung jener Struktur erreicht werden

\footnotetext{
+ Ebd., 154.

15 John B. Cobb, "A Whiteheadian Christology", in Process Philosophy and Christian Thought, hg. v. Delwin Brown u. a., New York 1971, 392.
} 
kann. «b $^{16}$ Diese Überzeugung impliziert Jesu universale Relevanz, ohne sie explizit zu beschreiben. Diese Aufgabe wurde im Detail durch John Cobbs Christ in a Pluralistic Age (1975) erfüllt.

Cobb weist auf die Absicht seines Hauptwerkes zur Christologie hin, wenn er sagt: "Dieses Buch schlägt vor, daß für uns Christus der Weg ist, der keine Wege ausschließt. «" Cobb versucht, diese Absicht durch eine Neuinterpretation der Logos-Christologie zu erreichen. Indem er die griechische Anschauung vom Logos als göttlichem Ordnungsprinzip mit dem johannëischen Verständnis des Logos als Gottes schöpferischer Kraft verbindet, die in Jesus Christus verkörpert ist, behauptet Cobb: "In der christlichen Tradition wird die transzendente Wirklichkeit, die in ihrer inkarnierten Form Christus genannt wird, als Logos bezeichnet. Christus ist der inkarnierte Logos. Der Logos ist das kosmische Ordnungsprinzip, der Bedeutungsgrund und die Sinnquelle. ${ }^{18}$ Die Interessen des Prozeßdenkens werden dann mit diesen Aussagen verbunden und der Logos wird zu "der geordneten Gegebenheit der relevanten Potentialität. Letztlich ist er das transzendente Ordnen von dem her die Neuheit Ordnung empfängt und das Geordnete Neuheit in der Welt «." Wie schwierig es ist, die griechisch-whitehead'schen theoretischen Konzepte mit der historischen Partikularität von Jesus als dem Christus zusammenzubringen, wird deutlich, wenn wir hören, daß ")Christus` deshalb ein Name für den Logos ist. $\aleph^{20}$ Christus ist nicht mehr der Name und kann sogar in Anführungszeichen gesetzt werden.

Der Logos ist nach Cobb die Macht der schöpferischen Umwandlung und das eine Prinzip aller bedeutsamen Ordnung. Inkarniert in allen Menschenwesen und aller Schöpfung gibt aber der Logos nicht allen die Gewißheit des Willens Gottes und die Autorität direkter Einsicht. Nur wenn er völlig gegenwärtig ist, macht er einen notwendigen Aspekt der Existenz aus und auch das Selbst als solches. $\mathrm{Da}$ in Jesus sein wahres Selbstsein durch den Logos konstituiert war, erklärt dies seine Gewißheit und Autorität. ${ }^{21}$ In Jesus vollzog sich eine entscheidende Inkarnation. Wiederum hören wir nur, daß sich etwas in oder mit ihm ereignete, aber nicht, daß Jesus selbst ein einzigartiges oder entscheidendes Ereignis war, d. h. Gottes Inkarnation.

Cobbs axiomatische Argumentationsweise wird wiederum deutlich, wenn er die Einzigartigkeit Jesu betrachtet. Zunächst gesteht er zu, daß, soweit man wisse, Jesus einzigartig ist. "[Doch] gibt es keine apriorische

\footnotetext{
16 Ebd., 398.

17 John B. Cobb, Christ in a Pluralistic Age, Philadelphia 1975, 22.

18 Ebd., 71.

19 Ebd., 75.

20 Ebd., 76.

${ }^{21} \mathrm{Vgl}$. ebd., $138 \mathrm{f}$.
} 
Grundlage, um zu entscheiden, ob andere auch an dieser Existenzstruktur teilhatten. Dies bleibt eine offene Frage. «22 Interessant sind nicht die Ergebnisse von Cobbs Argumentation, sondern seine Verfahrensweise. Warum sollten wir uns überhaupt a priori dieser Frage zuwenden und nicht vom historischen Kontext herkommend, vom Lebenswerk und Schicksal Jesu?

Die Gefahr, die in Cobbs Argumentationsweise liegt, wird am deutlichsten wenn er die christliche Hoffnung zur Sprache bringt. Er behauptet, daß »die Begegnung mit dem Buddhismus ein wesentlicher Schritt für den Westen sein kann, um sich von seiner Vorliebe für eine individualisierte personale Existenz als letztem Ziel zu befreien. ${ }^{23}$ Die Inkarnation, so sagt er, bewegt sich hin "auf eine Transzendenz der trennenden Individualität in einer vollständigeren Gemeinschaft mit anderen Menschen und allen Dingen. ${ }^{24}$ Cobb behauptet zu Recht, daß nach seiner Anschauung sich alle unsere eigenen Bilder des letztes Zieles auf diesen Punkt hin bewegen. Christus ist das Prinzip und die treibende Kraft der schöpferischen Umformung auf dieses Ziel hin. Aber Cobb zahlt einen hohen Preis dafür, daß er die universale Relevanz des Christusereignisses auf diese Art interpretiert. Zuerst sind wir erstaunt, wie wenig Aufmerksamkeit er dem Kontext schenkt, in dem Christus als der inkarnierte Logos bejaht wird, nämlich der Gemeinschaft der Gläubigen, die sich in historischen, kirchlichen Gruppierungen zeigt. Ohne die Gemeinschaft der Gläubigen wäre die Erinnerung an Christus schon lange in Vergessenheit geraten. Obwohl Cobb zweitens gewisse historische Verläufe in der Geschichte des christlichen Dogmas sorgfältig betrachtet, weist er das Ansinnen zurück, Jesus in dem ihm eigenen historischen Kontext zu verstehen (vgl. seine ausführliche Diskussion mit Wolfhart Pannenberg bezüglich der Auferstehung). Jesus wird somit mehr zu einem Prinzip statt zu einer historischen Gestalt.

Während Cobb sowohl die Kontingenz als auch die universale Relevanz Jesu Christi betont, interpretiert er beide mehr philosophisch als historisch. Deswegen sind sie mehr in axiomatischen Prinzipien verankert, d. h. im Prozeßdenken, als in den Verflochtenheiten der Geschichte. Die Einsichten, auf die sich diese Prinzipien gründen, sind der jüdisch-christlichen Tradition nicht völlig fremd. Aber diese Tradition dient mehr zur Illustration dieser Prinzipien, deren Wahrheit apriorisch vorausgesetzt wird, statt daß die Prinzipien dazu dienen, die christliche Erinnerung an Christus zu illustrieren und zu interpretieren. Für Cobb bedeutet universale Relevanz nicht die universale Bedeutung eines historisch kontingenten Ereignisses, d. h. des Lebens und Schicksals Jesu, sondern eines universalen

\footnotetext{
22 Ebd., 142.

${ }^{23}$ Ebd., 220.

${ }^{24}$ Ebd., 258.
} 
Prinzips, das auf ein historisches Ereignis angewandt wird. Diese Tendenz wird in der Christologie David Griffins noch deutlicher.

\section{David Griffin}

Schon in seiner Dissertation befaßte sich David Griffin mit der Christologie. Dieses Erstlingswerk wurde später zu der Veröffentlichung $A$ Process Christology (1973) ausgebaut. Thomas A. Idinopulos war vielleicht zu kritisch, wenn er die Arbeit Griffins dahingehend zusammenfaßte: "Dieses Buch ist im Grunde genommen eine logische Übung, deren Ziel es ist, analytisch zu demonstrieren, daß Whiteheads Verständnis des idealen Ziels ein gültiges philosophisches Konzept ist, um den sogenannten objektiven Pol von Gottes Offenbarung in Jesus Christus rational einsichtig zu machen und damit die Besonderheit und Einzigartigkeit Jesu Christi.«25 Man mag in der Tat überrascht sein, wenn man den Aufriß von Griffins Buch betrachtet. Nur ein Sechstel befaßt sich mit der eigentlichen Christologie, während der Rest auf dieses Thema hinführt, in dem es verschiedene Probleme des Offenbarungsverständnisses untersucht. Das heißt nicht, daß Griffin sein Untersuchungsobjekt leichtfertig bearbeitet hätte. Es deutet aber darauf hin, daß es für ihn eine Vielzahl notwendiger Vorüberlegungen gibt, bevor er das tatsächliche Thema bearbeiten kann.

David Griffin beginnt mit einer Suche nach dem historischen Jesus, um herauszufinden, wer Jesus wirklich war und was er tat. Er folgert, daß Jesus auf den Ruf Gottes antwortete, indem er im Sinne Gottes handelte und andere aufforderte, auch so zu antworten. Jesus muß im historischen Kontext Israels gesehen werden, da seine Botschaft die allgemeine Wirklichkeitsschau, wie sie sich in Israel herausgebildet hatte, voraussetzt. In diesem Kontext kann die Frage nach dem Neuen in Jesu Botschaft entschieden werden. Man erkennt dann, wie die verschiedenen Elemente seiner Botschaft verschiedenartig gewichtet werden. Die Veränderungen in der Gewichtung, die Jesus einführte, änderten die ganze Sicht der hebräischen Wirklichkeitserfassung. Griffin folgert daraus: $\bowtie$ Die Ziele, die Jesus gegeben wurden und von ihm wäbrend seiner aktiven Wirksamkeit verwirklicht wurden, waren solche, daß die grundlegende Wirklichkeitsschau, die in seiner Botschaft des Wortes und der Tat enthalten ist, der höchste Ausdruck von Gottes äußerem Charakter und Ziel wurde. ${ }^{26}$

$\mathrm{Da}$ der Inhalt von Gottes idealen Zielen für uns sich mit Zeit und Ort ändert und davon abhängt, was in einer bestimmten Situation das beste ist, versichert uns Griffin, daß ihre Verwirklichung nicht nur auf unsere eigene Initiative zurückgeführt werden kann. Die Besonderheit dieses Aktes muß

${ }^{25}$ Thomas A. Idinopulos, “A Critical Appraisal of Differing Process Christologies", Encounter 37 (Frühjahr 1976): 211.

${ }^{26}$ David R. Griffin, A Process Christology, Philadelphia 1973, 218. 
auch zum Teil Gott zugeschrieben werden. Griffin behauptet auch, es sei irreführend, bei Jesus von Gottes besonderem oder entscheidendem Akt zu sprechen, es sei denn, Gottes Tätigkeit in Jesus würde sich von seiner übrigen Tätigkeit unterscheiden. Doch »indem er Gottes partikulare Ziele für sich verwirklichte, drückte Jesu Gottes allgemeines Ziel für seine ganze Schöpfung aus. «" ${ }^{27}$ Dies bedeutet, daß die Tatsache, daß Jesu Gottes vollkommenster Akt war, nicht nur in Jesu Entscheidung begründet ist, Gottes Ziel für sich zu verwirklichen, sondern auch in Gottes Inhalt dieser Ziele. Jesus war als Gottes entscheidende Offenbarung auch Gottes Akt, ohne damit zu sagen, daß sich die menschliche Seite Jesu völlig passiv verhielt.

Indem er mit der Kontingenz der Wirksamkeit Jesu beginnt, daß er an diesem bestimmten Ort in Raum und Zeit Gottes entscheidende Tätigkeit ist, betont Griffin ihre universale Relevanz. Als Jesus uns Gottes Natur in der ihr eigenen Besonderheit enthüllte, enthüllte er auch Gottes Natur im allgemeinen. Da Tun und Sein gleichgesetzt werden muß, wurde uns damit Gottes Tun offenbart. Jesus enthüllte deshalb Gottes Natur und Tätigkeit in ihrer historischen Besonderheit und in ihrer allgemeinen universalen Gültigkeit. Von dem, was historisch sicherzustellen ist, folgert Griffin, daß es eine ontologische Möglichkeit gibt, daß »in dem Ereignis des Tuns Jesu Gott in vollkommenster Weise seinen Charakter und sein Ziel ausdrückt und damit seine Handlungsweise. ${ }^{28}$ Deshalb kann Jesus zu Recht der Christus genannt werden, und der Glaube an Jesus, der im christlichen Glauben wurzelt, ist sachgemäß. Jesus war Gottes vollkommenster Akt der Selbstenthüllung und kann deshalb zu Recht als Gottes entscheidene Offenbarung verstanden werden.

Wenn wir versuchen, Griffins Ansatz zu beurteilen, bemerken wir, daß er sich nicht auf einen Dialog mit anderen Religionen einläßt, die auch behaupten, eine besondere Art von Gottes Selbstoffenbarung zu beinhalten. Für Griffin ist Jesus Gottes vollkommenster Akt, durch den in einer unübertrefflichen Weise eine neue Schau der Wirklichkeit ausgedrückt wurde. Da Griffin sich mit Gottes Verhältnis zu Jesus befaßt, erhält der einzigartige Pol von Jesu historischer Besonderheit adäquate Aufmerksamkeit. Aber was bewirkte Jesus?

Nach Griffin sind wir durch Christus und seit dem Christusereignis befähigter, uns mit Gottes Zielen zu identifizieren. Zumindest in Annäherung ist für uns in dieser Welt Ganzheit, Güte und Ruhe erreichbar. Doch die universale Relevanz Christi bleibt durch diese Welt begrenzt, durch das Raum-Zeit Kontinuum, in dem wir leben. Es gibt nichts wesentlich Neues unter der Sonne, sondern nur neue Konfigurationen des schon bisher Möglichen. Sogar die buddhistische Anschauung des Nirwana würde über 
diese Begrenzung hinausweisen. Der Grund für die universale Relevanz Jesu, die in unserer gegenwärtigen Welt ihre Begrenzung findet, liegt mit darin, daß Griffin Jesu Auferstehung (Erscheinungen) als nicht konstitutiv für Jesu Person erachtet. Griffin behauptet damit konsequent, daß der christliche Glaube möglich sei unter Absehung von »dem Glauben an Jesu Auferstehung im besonderen und dem Leben jenseits des körperlichen Todes im allgemeinen. « $^{29}$ Während John Cobb versucht, den christlichen Auferstehungsglauben mit der buddhistischen Anschauung des Nirwana zusammenzubringen, klammert Griffin ersteres als eine Option ein. Obwohl wir zugestehen, daß die historische Kontingenz Jesu tatsächlich mit seinem Tod endet, kann seine universale Geltung nicht als allumfassend behauptet werden, wenn man nicht auch Christi Auferstehung berücksichtigt.

In klassisch liberaler Weise konzentriert sich Griffin auf Jesu Verhalten und Verkündigung, wobei er versucht, ihn in den Kategorien des Prozeßdenkens zu verstehen. Bei diesem Vorgehen übersieht Griffin, daß wir in den Evangelien Passionsgeschichten mit langen Einleitungen über Verhalten und Verkündigung Jesu begegnen. Infolgedessen bietet er statt der Realität des Todes und der Begrenzung, die sich in ihm zeigt, die liberale Verheißung eines liebenden und sich um uns bemühenden Gottes. $\mathrm{Da}$ dieser Gott eng mit der Struktur unserer Welt verbunden ist, muß die universale Geltung Jesu Christi, die sich davon ableiten läßt, auch die Kennzeichen der Begrenzung (Griffin) und Relativierung (Cobb) tragen. Vielleicht hatte Lewis Ford recht, wenn er behauptete: "Die Prozeßtheologie sollte die Frage nach Gottes ewiger Natur und Zweck der Metaphysik überlassen, wohin sie sachgemäß gehört, und ihre Energien auf dieses Problem konzentrieren: Was wollte Gott für die Menschheit in Leben, Tod und der Auferstehung Jesu bewirken? Auf diese Weise könnten wir eher und präziser artikulieren, was denn das sachgemäße christologische Ziel sein sollte. ${ }^{30}$

${ }^{29}$ Ebd., 12. Vgl. auch seine etwas ausführlicheren Ausführungen zu diesem Thema in seinem Anhang, "Theodicy and Hope for a Future Life", in David R. Griffin, God, Power, and Evil: A Process Theodicy, Philadelphia 1976, $312 \mathrm{f}$.

${ }^{30}$ Lewis S. Ford, "The Possibilities for Process Christology", Encounter 35 (Herbst 1974): 294. Lewis Ford selbst befaßt sich zu Recht mit der Auferstehung Christi, um Christi Universalität zu behaupten. Aber auch er interpretiert die Auferstehung als das Hervortreten des Leibes Christi, d.h. der Kirche, wobei er vernachlässigt, daß das historische Geburtsdatum der Kirche Pfingsten und nicht Ostern war. Nach Ford wird der einzelne durch Christi Auferstehung in seinen Leib einbezogen, wodurch er seine Individualität verliert, statt zu einer ihm gleichen Auferstehung befähigt zu werden. Wir bemerken auch bei Ford, obwohl wesentlich weniger als bei Cobb und Griffin, daß er versucht, die geschichtliche Individualität Jesu mit den begrenzten Möglichkeiten der Denkstrukturen Whiteheads aufzuzeigen. - Vgl. sein bedeutsames Buch The Lure of God. A Biblical Background for Process Theism, Philadelphia 1978, bes. 71-80. 
Wie jedes philosophische System in der Geschichte der christlichen Theologie, ist die Prozeßphilosophie eine theoretische Abstraktion, die als solche den Verschlungenheiten der Geschichte nicht gerecht werden kann. Wie die traditionelle Christologie von oben, konstruiert die Prozeßchristologie ein universales Prinzip (kosmische Liebe, schöpferische Umformung, ideales Ziel), das auf den Jesus der Geschichte angewandt und von daher universalisiert wird. Doch die Bewegung sollte von dem besonderen (lokale Geschichte) zum Universalen (universale Geschichte) sein. Da wir das Partikulare nicht voraussetzungslos erfassen können, müssen wir immer unvermeidbare Voreingenommenheiten als solche erkennen und selbstkritisch jede Idealmethode untersuchen, die wir gefunden zu haben scheinen. Jedes Wahrnehmungswerkzeug muß durch andere Werkzeuge korrigiert werden, damit es einen genügend großen Teil von Gottes letztlicher Selbstoffenbarung erfassen kann, so daß wir beginnen können, ihre universale Bedeutung zu erkennen. Nur streng eklektisch kann ein Whitehead'sches Gerüst die historische Individualität von Jesus insoweit erfassen, daß Jesus Christus wirklich als die universale Hoffnung für die Menschheit beschrieben werden kann, die unsere Begrenzung und Zerbrochenheit durch ein neues Beginnen aufhebt.

\section{SUMMARY}

Process theology has made a modern attempt to ground Christian theology on a rational metaphysics. The question emerges, however, whether the universality of process philosophy can adequately account for the historical contingency of the Christian revelation.

The issue of Christology offers a poignant test case for examining this tension between historical contingency and universality in the thought of three process theologians: Norman Pittenger, John B. Cobb, and David Griffin. Following an extensive exposition of the thought of these three theologians, the conclusion is drawn that process philosophy on the basis of its level of abstraction and universal categories is limited in its ability to conceptualize the historical particularity of God's self-disclosure in Jesus Christ. 